\title{
Germanica
}

\section{Modellfall in der Kakastrophe der Gegenwart : Die «Wiedervereinigung » bei Johannes Mario Simmel}

Modellfall in der Kakastrophe der Gegenwart : la « Réunification » chez Johannes Mario Simmel

\section{Friedbert Aspetsberger}

\section{OpenEdition}

\section{Journals}

Édition électronique

URL : http://journals.openedition.org/germanica/2328

DOI : 10.4000/germanica.2328

ISSN : 2107-0784

\section{Éditeur}

Université de Lille

\section{Édition imprimée}

Date de publication : 30 décembre 1999

Pagination : 27-36

ISBN : 9782913857018

ISSN : 0984-2632

\section{Référence électronique}

Friedbert Aspetsberger, « Modellfall in der Kakastrophe der Gegenwart : Die « Wiedervereinigung » bei Johannes Mario Simmel », Germanica [Online], 25 | 1999, Online erschienen am: 28 Januar 2014 abgerufen am 06 Oktober 2020. URL : http://journals.openedition.org/germanica/2328 ; DOI : https:// doi.org/10.4000/germanica.2328

Ce document a été généré automatiquement le 6 octobre 2020.

(c) Tous droits réservés 


\title{
Modellfall in der Kakastrophe der Gegenwart : Die «Wiedervereinigung » bei Johannes Mario Simmel
}

\author{
Modellfall in der Kakastrophe der Gegenwart : la « Réunification » chez Johannes \\ Mario Simmel
}

Friedbert Aspetsberger

\section{I}

1 Johannes Mario Simmel, «Verfasser demokratischer Gebrauchsliteratur $»^{1}$, greift zu aktuellen Stoffen und Problemen, um seine Absichten zu realisieren : die literarische Unterhaltung, politische Aufklärung und Bildung der Leser.

21965 erschien der Roman "Lieb Vaterland magst ruhig sein », der die Berliner Mauer anschaulich-sinnlicher Höhepunkt der Auseinandersetzung zwischen Ost und West am Beispiel der beiden 1949 gegründeten « deutschen » Staaten - zum Gegenstand hat. Das Buch, das die politischen Verhältnisse in gängigen Genres wie der Spy story, aber auch mit Mustern nach der Kanonliteratur, z. B. Alfred Döblins Roman «Berlin Alexanderplatz » (1929), darstellt, erreichte bisher eine Auflage von 4,2 Millionen. Den Abriß der Berliner Mauer bzw. die « Wiedervereinigung » behandelt der Roman « Auch wenn ich lache, muß ich weinen» (1993). Bisher wurden 1,2 Millionen Exemplare verkauft. Bei einer Gesamtauflage der Werke Simmels von 73 Millionen darf man einerseits annehmen, daß er sein Publikum auch in dem, was es politisch verträgt, zu kalkulieren weiß, anderseits aber auch, daß es ein großes Publikum für eine aufklärende « demokratische Gebrauchsliteratur » gibt; denn an Simmels moralischem Engagement, z. B. am strikten Antifaschismus seiner Werke, besteht so wenig Zweifel wie am perfekten Handwerk des aus Wien stammenden Erfolgsschriftstellers (geboren 
1924), der lange in Frankreich lebte und dessen mehr als zwei Dutzend Romane in dreißig Sprachen übersetzt wurden ${ }^{2}$.

\section{II}

«Auch wenn ich lache, muß ich weinen » stellt die « Wiedervereinigung » nicht isoliert dar, sondern als Teil der Scheiße bzw. der "Kakastrophe » $(391)^{3}$ der gegenwärtigen Weltpolitik und Weltwirtschaft. Beide werden als psychische Ausscheidungen der Herrschenden gesehen und im Bild der Körper-Ausscheidungen gefaßt. Die FäkalMetapher führt den Leser durch den Roman, in dem die politischen Verhältnisse an den hauptsächlichen Welt-Krisenherden exemplarisch zur Anschauung gebracht werden: Nach dem deutschen Beispiel folgen Rußland, Irak und Israel, Bosnien und, wenn auch in gemilderter Form, die USA. Die Menschenverachtung steht der - sehr gebrochenen Hoffnung auf eine Veränderung zum Bessern gegenüber. Wird die eine im perfekt verbergenden Design von Sanitär-Artikeln dargestellt, so die andere in Gestalt von Konstruktionsplänen für ein "Öko-Klo», mit dem die Ausscheidungen recyclet und brauchbar wieder in den Welt-Bestand integriert werden könnten. Ob das « Öko-Klo » vom Helden des Romans, dem Installateur Mischa Kafanke, bis zu Prototypen entwickelt - je in Serie gehen wird, bleibt am Ende des Romans offen.

Das Closett ist also Allegorie der beschriebenen politischen Verhältnisse. Simmel setzt Allegorien, seit dem Mittelalter hochentwickelte, didaktische Textmaschinen, häufig $e^{2 i n}{ }^{4}$. Die anscheinend « realistischen » Mittel seines Zeit-Romans sind immer moralisch Sprechende Bilder. Die beschriebenen Sanitär-Artikel halten die großen Bedeutungen von Welt-Frieden und Welt-Krieg in der reichen Romanhandlung anschaulich präsent. Sie sind in diesem Sinn didaktischer Teil der maniera grande des aufklärend-politischen Erfolgsschriftstellers. Simmel klickt aber auch zahlreiche andere Mittel auf der MenueLeiste zur Romanherstellung an, auf die im folgenden fallweise eingegangen wird. «Auch wenn ich lache, muß ich weinen » ist sein zweiundzwanzigster Roman ${ }^{5}$.

III

5 Simmel ist in der Handhabung des Vulgären als repräsentatives literarisches Bild erfahren. Seine satirische Darstellung des deutschen "Wiederaufbau »-Wunders in «Hurra wir leben noch» (1978) bediente sich einer sexuellen Metapher, der « Hasenpfote ». Der Held des Romans mit dem sprechenden "Macher »- und MachoNamen Formann trägt sie immer in seinem Hosensack, fühlt sie sich bewegen, hüpfen und zittern, und «massiert » sie in Todesangst. Er wird mit seinem Penis (« Pimmel ») ident gesetzt ${ }^{6}$. Simmel läßt es beim Ausbau dieser Metapher in Szenen des Alltags nicht an Deutlichkeit fehlen : Formann gründet Hühnerzuchten zur Produktion von « Eiern ». Sie werden auch in die DDR exportiert, doch enthalten diese Kisten nur eine Lage Eier, der Raum darunter wird mit Porno-Material gefüllt. « Formann » produziert auch EierLikör und vertreibt ihn mit einem Bus-Verkaufssystem bis in den hintersten Winkel der Bundesrepublik: das Wiederaufbau- "Wunder» erscheint bei simmel als scrotaltestikulär verursachter Wiederaufbau-Rausch pubertärer Allmachtsphantasien «Formanns» bzw. seiner Aufsteiger-Generationsgenossen. Der scharf satirische Unterhaltungsroman erreicht eine Auflage von über 3,7 Millionen. Er prognosziert der «Wiedervereinigung » nichts Gutes aus dem Westen. 
Simmel bleibt in « Auch wenn ich lache, muß ich weinen » auf der Linie von « Hurra » : Busse aus dem Westen vertreiben mit größtem Erfolg Porno-Material auch in der ehemaligen DDR-Kleinstadt Rotbuchen, in der Mischa Kafanke sein Installationsgeschäft unterhält. Mischa läßt sich überreden, beim Aufbau eines Vertriebs von « geschmacksaktiven » Präservativen mitzutun. Zwar sind die «neu für die neuen Länder » und scheinen ein sicheres Geschäft, « denn gefickt wird immer [...], unter jedem Regime" (137), doch liefern die Erzeuger im Westen zu wenig in der Geschmacksrichtung, die die "Ossis» bevorzugen. Für Mischa endet das Geschäft damit, daß er in « Oderstadt » bei rassistischen Auseinandersetzungen schwer verletzt wird. Simmel typisiert den Zusammenhang von Sex und Politik, indem er auch ein anderes Sex-Geschäft Mischas, das ideologisch charakteristischer ist, mit einer brutalen Prügelei enden läßt. Simmel baut sie auf ein Porno-Bild, die Großaufnahme einer nackten Frau im Bett, auf : « nackt und herrlich anzuschauen, alles, alles, die Brüste, die Schenkel, der flache Bauch, der Schoß, das schöne Gesicht, die weiche, glänzende Haut [...] » (116). Das Bild demonstriert Mischas pubertäre Phantasie und wird in der Realität entsprechend destruiert. Nach einem Coitus von gewaltiger Intensität und Dauer wird Mischa von der Schönen, Lilly, und ihrem Freund blutig geschlagen und bestohlen ${ }^{7}$. Lilly, ihr Freund, Mischa und andere hatten gemeinsam einen schwungvollen Handel aufgezogen : sie verschleudern mit bestem Gewinn, was die DDR an Zeichen für ihr ideologisches (sozialistisches) Kapital aufwies und was sie in ihrer politischen Praxis selber verschleuderte. Vor dem Brandenburger Tor verkaufen Mischa \& Co. die DDREhrenzeichen und Orden, ziehen aber auch einen Abbruchdienst auf, der sie mit rasch bemalten Mauerstücken versorgt. Denn die Berliner Mauer reicht für den Bedarf an Ideologie-Relikten im siegreichen Westen nicht aus : der schon firmenmäßige Export der Mauerstücke in die USA wird noch gewinnträchtiger, als für die Mauer-Trümmer Abbruchhäuser der New Yorker Bronx verwendet und mit dem Zertifikat «THIS IS TO CERTIFY THAT THIS IS A PIECE OF THE ONE AND ONLY BERLIN WALL » (124) versehen werden. Die ideologischen Zeichen des DDR-Regimes verwandeln sich zuerst in « Souvenir »-Waren und gehen dann ganz im Schein-Zertifikat der westlichen Werbe-Welt auf. Dem entspricht die Vergrößerung des cleveren DDR-Mädchens Lilly zum Vamp-Sex-Bild, dem entspricht aber auch die Desillusionierung Mischas, der an dieses Bild glaubte. Die humane Desillusionierung in der neuen Welt des Westens wird nicht nur individuell an Mischa gezeigt, sondern vor allem in der metaphorischen Ebene an den Inhabern von KZ-Ehrenzeichen, die ihre Orden nun den Verkäufern aufdrängen : sie verkaufen zeichenhaft - ihr Leben in den Bedeutungen eines humanen Kontextes, in dem sie sich ursprünglich gewürdigt meinten.

\section{IV}

7 Simmel eröffnet den Roman melodramatisch mit der Zerstörung eines Zukunftsbildes. Damit wird einerseits ein kritisches Bild der DDR gegeben, anderseits die «Abwicklung » der Erbschaft, die die DDR für die BRD darstellt, negativ beurteilt :

Zwei Kinder in der Kleinstadt Rotbuchen nahe Berlin, die unsterblich ineinander verliebt sind, werden in den Selbstmord getrieben, weil das Gerücht ihre Eltern der Mitarbeit beim Staatssicherheitsdienst der DDR beschuldigt. Zwar stellt sich heraus, 
daß die Beschuldigung nicht stimmt, doch sind die Kinder inzwischen geflohen und bringen sich um - in den schmutzigen und verrotteten Sanitärräumen einer verlassenen Kaserne. Simmel setzt Kinderrollen auch in anderen Romanen ein, um die Schuld der Erwachsenen gegenüber der Zukunft zu demonstrieren, etwa den Knaben Hansi in «Ich gestehe alles » (1953), der psychisch gestört ist, oder das Mädchen Patty in «Bitte laßt die Blumen leben » (1983), das hinkt, doch ging er nie so weit, die Kinder sterben zu lassen. Der Tod der Kinder als Ouverture des Romans beschreibt sowohl die Stasi-DDR und als auch ihre « Abwicklung » durch den Westen human ruinös.

9 Im melodramatischen Kinder-Bildfenster erscheint auch das Bild des Protagonisten Mischa Kafanke zum ersten Mal und wird dann herausvergrößert. Mischa wird mitschuldig am Tod der Kinder, weil er sie der Polizei anzeigt, als sie sich zufällig in sein Installationsgeschäft flüchten. Er ist, im Sinn der Grundmetapher des Romans, schon zu sehr Teil der egoistischen Waren-Welt, die sich in seinem Laden bereits breit gemacht hat: der "VEB [volkseigene Betrieb] Sanitas", der Mischa bisher mit zufriedenstellenden, aber nicht schönen Waren belieferte, wurde "abgewickelt», $d$. h. zugesperrt, der Westen, hier beschrieben als Großbetrieb "Clo-o-Form », plaziert mit Nachdruck seine schön designten Geräte der Fäkalbeseitigung auf dem neuen Markt der liquidierten DDR. Unter dem Werbespruch « NUN ENDLICH SCHÖNER LEBEN » stehen der hilflose Mischa und die beiden hilflosen Kinder zwischen den «noch nie gesehene[n] Klos », deren Namen jeden Stuhlgang als (teils touristische) Erlösung im Schönen verkünden :

Blitzende Klosettschüsseln mit Deckeln und seltsamen Aufbauten, die allesamt Namen haben, Namen! Sie stehen auf Täfelchen vor ihnen: «Moderna », « Capella », «Vienna », «Magnum », «Piano », «Grangracias », «Opera », «Marina », «Closomat Rio», «Closomat Lima» «Closomat Samoa »... Das hältst Du im Kopf nicht aus, Klos in Weiß, in Rosa, Blau, Hellgrün, Gelb, Schwarz, verziert, vergoldet... ! (23).

10 Mischa, dem die Firma «Clo-o-Form» diese Muscheln auf Credit in seinen in DDRZeiten gutgehenden Laden gestellt und eben diesen Laden samt Grundstück als Sicherstellung genommen hat (und ihm beides wegnehmen wird), kommt mit den Kindern "in fließendem Übergang auf die modernen Klos zu sprechen", statt sich ihrer Sorgen anzunehmen (34). Auch Mischa hat sich also vom « NUN ENDLICH SCHÖNER LEBEN » « abwickeln » lassen.

11 Er wird freilich nicht negativ gekennzeichnet, sondern in seiner menschlichen Verwirrung und seiner politischen Unbrauchbarkeit, als Opfer der Verhältnisse und seiner Herkunft. Noch im melodramatischen Bereich steht seine rührende Charakterisierung als "Basset Hound" nach dem "Standardwerk "Kynos-Atlas. Hunderassen der Welt" ", das die Kinderliebe und die " großen melancholischen Augen des Basset » zur captatio benevolentiae des Lesers betont : « Alle echten Basset-Freunde sind dieser Rasse verfallen »(28). Die aus dem Hunde-Atlas einmontierte Passage ist bedeutungsvoll, da Mischa tatsächlich eine besondere «Rasse » ist: Der noch nicht Dreißigjährige ist der Sohn eines Sowjet-Offiziers jüdischer Herkunft und einer deutschen protestantischen Pfarrerstochter. Dadurch gehört er nirgends von vornherein und selbstverständlich dazu und wird - in mehreren Amtsvorgängen, bei Vorsprachen beim Vorsitzenden der israelitischen Kultusgemeinde, beim 
evangelischen Superintendenten und im weitern im ganzen Roman, auch auf dem Schauplatz Israel - als der amtsideologisch bzw. "politisch" nie ausreichend eindeutige (eigentliche) Mensch charakterisiert. Seine - und jede - « Mischung " paßt nirgends, wenn es - wie bei Sanitärgegenständen und Ideologien - um "Reinheit » geht. Mischa als «Basset » ist also nicht zu unrecht rührend dargestellt. Er ist das Bild des Menschen schlechterdings und damit Bild der Verfolgungen. Mischa spürt - nach der jüdischen Zeitrechnung - den sechstausendjährigen Wind der Gefährdung, der ihn in seine Odyssee treibt. Er wäre auf humane Prinzipien in der Gesellschaft angewiesen, nach denen aber keine der Gesellschaften, die er kennenlernt, sich organisiert. Das rüh rende Moment des « Basset Hound » fungiert also auch als politisch-satirisches.

\section{V}

Das wiedervereinigt "deutsche » Leben Mischas endet so doppelläufig wie es der realistisch/metaphorisch doppelläufig geführten Romanhandlung entspricht. Zum einen kann Mischa den Credit der Firma «Clo-o-Form» nicht bezahlen, weil er aufgrund der Arbeitslosigkeit nichts verkauft ; er sitzt "Bis zum Hals in der Kacke » (75). Zum andern wird er, im Rahmen seiner Beteiligung am scheiternden PräservativGeschäft, in die Exzesse von « Oderstadt » verwickelt und dabei auch körperlich schwer verletzt. Simmel kombiniert sein metaphorisches Verfahren stets mit einem zeitgeschichtlich-dokumentarischen. In dieser Schicht des Romans gibt er $\mathrm{zu}$ «Oderstadt » eine Fußnote : « Eine Stadt mit diesem Namen gibt es nicht. Hingegen gibt es die Stadt Hoyerswerda. Die im folgenden geschilderten Ereignisse haben sich, nur zeitverschoben, genau so in Hoyerswerda ereignet " $(169)^{8}$. Die Exzesse werden zwar von radikalen Neonazis veranstaltet, aber auch "Frauen in Morgenmänteln, die Säuglinge auf dem Arm halten, junge Männer und alte Männer » beklatschen sie, und weder Polizei noch Politiker treten ihnen ausreichend entgegen (175). Der Innenminister spricht von der « Zwischenlösung » einer Umsiedlung, die noch nicht die « Endlösung » sei. Mischa, der « Mischling », « hat Todesangst » (180 f.).

Er will - er muß - auswandern. Seine Hoffnung richtet sich auf Dimitrowka, das Heimatdorf seines Freundes Leon Petrakow, Leutnant der russischen Armee, deren Versorgung zusammengebrochen ist. Leon schlägt sich schlechter als recht - aber in Art des Schelms Thomas Lieven aus Simmels Roman «Es muß nicht immer Kaviar sein » (1960) souverän - durch.

Nach der «dokumentarischen » Darstellung nach Medienberichten, die Simmel für Oderstadt/Hoyerswerda anwendet, läßt er für die Hoffnungen, die Mischa auf Dimitrowka setzt, ein « Märchen » folgen.

Das "Märchen » beginnt mit einem kosmischen Bild. Mischa besucht mit Irina, der Schwester seines Freundes Leon Petrakow, das Moskauer Planetarium. Er sieht sie als seine Frau. Im Anblick der Sternenwelt entwerfen Mischa und Irina - ein sprechender Name wie seiner - ihre Lebenshoffnungen. Vielleicht zitiert Simmel mit der Sternenwelt des Planetariums das Bühnenbild von Robert Musils « Die Schwärmer " . Sicher zitiert er mit der Sternenwelt ein berühmtes Bild des «Sozialistischen Realismus», nämlich den Beginn von Erwin Strittmatters DDR-Bestseller «Ole 
Bienkopp» (1963) ${ }^{10}$. Die kosmische Ouverture beleuchtet dort das Leben des Protagonisten und sein Scheitern durch die "real existierenden » Funktionäre. Bei Simmel ist es der Apparatschik Kotikow, der Mischas Versuch, in Dimitrowka ein Leben aufzubauen, beenden wird. Simmel hält die Hoffnung aber in Irina, die Mischa nach den USA folgen wird, aufrecht. In Irina wird Mischas Sex-Leitbild (Lilly) zum einem asexuellen Frauenbild gewandelt. Sie trägt eine Brille mit sechs Dioptrien links und sieben Dioptrien rechts. Nimmt sie die Brille ab, kann sie, von der Realität unbehelligt, ihrer Sternenwelt nachhängen : « Eine Welt, wie sie sein sollte, ohne Hass, voller Liebe, denn die einzige Hoffnung der Menschen ist die Liebe, sagt Irina, und die Liebe zum Leben ist die Quelle aller Liebe, und so sieht sie dann nur glückliche Menschen ». (99)

Mischa hat also das «wiedervereinigte » Deutschland mit einer Anspielung auf ein Meisterwerk der DDR-Literatur verlassen. Er verknüpft auch im weitern seine Hoffnungsbilder mit dem Sozialismus. Er zitiert Günther Grass' Wort von der «Konföderation» der beiden Staaten als der besseren Lösung gegenüber der abwickelnden «Wiedervereinigung » (166) und beruft sich Irina gegenüber auf einen Artikel von Robert Little, der in « Die Zeit » veröffentlicht worden sei und der ebenfalls die Hoffnungs- bzw. Märchenstruktur des Sozialismus als einer bessern Welt betont : «Denn hinter der Grundidee des Kommunismus stand die Vision von einer optimistischen, idealistischen Menschheit : der Gedanke, daß Männer und Frauen ihre besten Gaben der Gesellschaft zur Verfügung stellen und dafür bekommen, was sie zum Leben brauchen" (280). Doch sind das seltene und geschickt in die Handlung integrierte Ausflüge ins Begriffliche. Viel entscheidender ist die Ebene der Alltagsbemerkungen, die Simmel für seine Leser durch Mischa gibt (« das mit der Wiedervereinigung ist keine gute Idee gewesen »;166) oder aus den Sprüchen und Witzen der Kleinen Leute in den Roman zitiert. Freilich ist auch diese Form der Beurteilung im Rahmen der Romanhandlung so sarkastisch wie die mit dem Sarkasmus getroffene Lage erschreckend ist : "Was die sozialen Verhältnisse im Osten betrifft, so will ich Dir nur einen von vielen Witzen mitteilen, die bei uns erzählt werden - den kürzesten und bösesten : Treffen sich zwei Ossis bei der Arbeit. Aus. Schluß. Das war schon die Pointe » (279).

Entscheidend aber ist, daß Mischas Arbeitsfähigkeit als Öko-Klo-Konstrukteur, das heißt Entsorger der Welt-Scheiße, aus seiner DDR-Identität herstammt. Zwar durfte er nicht studieren, hat sich aber in den vorzüglichen - das wird mehrfach im Roman wiederholt - Bibliotheken der DDR so weit fortgebildet, daß er zum Erfinder eines ÖkoKlos werden konnte. Das ist weniger auf der realistisch-dokumentarischen als auf der metaphorischen Ebene des Romans zu lesen : Mischa ist mit der Konstruktion eines "Öko-Klos» auf der Suche nach Konstruktionsformen für den Frieden. Seine Ausbildung bzw. seine Selbstbildung in der DDR ermöglichen es ihm, schließlich in den USA Fuß zu fassen. Die satirische Voraussetzung dafür ist aber, daß sich das Menschenbild der Werbung verändert. Die Hoffnung auf die Realisierung seiner Friedenskonstruktion «Öko-Klo" ist also weiterhin von sehr fragilen Bedingungen bestimmt, bezieht anderseits nun auch den « Dritten Weltkrieg » der Werbung ein (523 ff.). 


\section{VII}

dem Moskauer Bahnhof begegnet Mischa einer grotesk gezeichneten Wahrsagerin, die ihm weissagt, was er selber kurz vorher einem Knaben erzählt hat, der augenscheinlich mit der Wahrsagerin unter einer Decke steckt. Sie verweist Mischa auf die Hoffnung als einzige Rettung aus seiner Misere. Aber die Wahrsagerin verkörpert (literarisch) auch die Lösung der russischen, das heißt der Mafia-Verhältnisse : Simmel deckt sie als Jean Giraudoux' " La Folle de Chaillot » auf und macht damit zugleich sein Romankonzept als "Mischung» aus Heiter-Groteskem, Romantisch-Märchenhaften und aus Kritik am westlichen Materialismus deutlich. Vergleichbar löst er dem Leser die widersprüchliche Situation in Israel (wo wohlmeinende Atomforscher einen Teil der Atom-Anlage von Dimona für die Erzeugung von Mischas Öko-Klo umzurüsten bereit sind, was aber verhindert wird und zu seiner Abschiebung führt) mit der Zitation von Jean-Paul Sartres « Les Séquestrés d'Altona ».

\section{VIII}

Das Finale des Romans in den USA hält die Spannungen, die den Text des Romans in seiner Vielfalt kennzeichnen, in vollem Umfang aufrecht. Mischa liest, umgeben von den Rassenunruhen in Los Angeles, Art Spiegelmans «Mouse. A Survivor's Tale » (1986), eine KZ-Darstellung in der Mäuse-Katzen-Schweine-Form des Comics, also der (nur anscheinenden) Banalisierung einer Menschheitsbedeutung in einer "primitiven» Form. Dem entspricht, wie an den Closetten ausgeführt, Simmels Vorgehen. Dem entspricht aber auch die Hoffnung Mischas, nun aufgrund eines veränderten Menschenbildes der Werbung die Produktion des Öko-Klos durch die Firma AMSAN beginnen zu können. Der Paukenschlag im Finale besteht darin, daß die amerikanischen Prototypen nicht funktionieren, weil die Amerikaner nicht über die für die Zersetzung der Exkremente nötigen Filter zur Isolierung der extrem aggressiven Bakterien verfügen, die Mischa in seine eigenen Prototypen in der DDR eingebaut hatte: sein Öko-Klo stellt sich in diesem Sinn als ein Produkt der Forschung der sozialistischen Bruder-Länder heraus, das mit Hilfe der Filter des «VEB Biotechnik Lyssenko » funktionierte. Er muß - nachdem er die Reise Irinas nach Amerika gesichert hat - in die ehemalige DDR zurück, um das Filter-Know-how des "abgewickelten » «VEB Biotechnik Lyssenko» (auf der gesellschaftlichen Ebene des Romans den Sozialismus) in die USA zu holen. Der Roman ist auch eine nach rückwarts gewandte Utopie einer besseren Welt. 


\section{NOTES}

1. Günther Rühle: Die Welt des Johannes Mario Simmel. In: Frankfurter Allgemeine Zeitung, 26.9.1970. Simmel hat dieser Formel in Interviews zugestimmt. Vgl. Peter Huemer : Im Gespräch. J. M. Simmel. 21.1.1988. ORF 1.

2. Vgl. Ute Weinmann: Die Rezeption J. M. Simmels in Frankreich. In : Johannes Mario Simmel lächelt. Innsbruck Wien : Studienverlag 1999, S. 127-150.

3. Die Seitenzahlen im Text beziehen sich auf die Taschenbuchausgabe des Romans (München: Droemer Knaur 1995).

4. Walter Grond sieht den Einsatz solcher Formen bei Simmel als avantgardistisches Moment. Vgl. W. G. : Die Verspätetheit sogenannter hoher Literatur. In: J. M. Simmel lächelt, a. a. O., S. 17-26.

5. Aus den Studien zu Simmels literarischer Technik seien hier nur erwähnt: Wolfgang Langenbucher (Hrsg.) : «Berichte über die Zeit, in der ich lebe... » J. M. Simmels und seine Romane. München Zürich : Droemer Knaur 1978. - Kurt H. Eissfeldt : Fatalismus und Hoffnung. Frankfurt/M. : Lang 1988. - Monika Schmiedt-Schomaker: J. M. Simmel als Bestseller-Autor. Königstein/Ts. : Forum Academicum 1979. - Katharina Schlicht: Die Figur des Erzählers bei J. M. Simmel. Marburg : Hitzeroth 1989.

6. J. M. Simmel : Hurra wir leben noch. München: Droemer Knaur 1981 (Taschenbuchausgabe), S. 302.

7. Simmel liebt die Wiederholung gleicher Bilder, um ihre Bedeutung dem Leser faßlich zu machen. Im Mafia-Moskau fällt Mischa auf das Super-Vamp-Bild der ehemaligen amerikanischen Agentin Melody Lyndon hinein, die er mit Hilfe von Potenz-Pillen und Sex-Prothesen zu befriedigen sucht. Sie wird auf ihn angesetzt, als man seine Öko-Klo-Konstruktionspläne für Pläne zur atomaren Wiederaufbereitung hält: einerseits wird seiner Beschäftigung mit der (politischen) Scheiße also bedeutende Sprengkraft zugebilligt, anderseits wird er von Friedensstifter (« Öko-Klo »-Erfinder) zum Kriegsforscher entidentifiziert.

8. In der «Dokumentation» der rassistischen Exzesse wiederholt Simmel die Verwebung von Sexualität und Politik wie bei Lilly und Melody Lyndon. Sie hat hier die Gestalt der 16jährigen « hübsche[n], üppige[n] Elfi mit den Hotpants » : «[... ] diese Elfi, die schmeißt jetzt aus ihrem Wohnzimmerfenster Flaschen auf die verfluchten Ausländer und ist außer sich vor Freude, als sie einen trifft und der zu Boden geht. Sie heult auf, als hätte sie einen Orgasmus, und so was ähnliches hat sie wohl auch ». (175).

9. Im 2. Akt schreibt Musil für seine «vulkanischen » Menschen, in denen " ein Rest von der Schöpfung her » noch nicht fest geworden ist, vor, daß über den Zimmer-Kulissen " und an manchen Stellen sogar zwischen den Büchern Sternennacht" sichtbar sein müsse (R. M.: Gesammelte Werke. Hrsg. von Adolf Frisé. Reinbek bei Hamburg 1978, Bd. 2, S. 399, 348).

10. «Die Erde reist durch den Weltraum. Der Mensch sendet eiserne Tauben aus und harrt ungeduldig ihrer Heimkehr. Er wartet auf ein Ölblatt von Brüdern auf anderen Sternen. [/] Was ist ein Dorf auf dieser Erde? Es kann eine Spore auf der Schale einer faulenden Kartoffel oder ein Püntchen Rot auf der besonnten Seite eines reifenden Apfels sein.» Der Schluß des Romans wiederholt den zweiten Absatz (Berlin Weimar : Aufbau 18.1975, S. 7, 217, 428). 


\section{RÉSUMÉS}

Die «Wiedervereinigung " wird in Simmels Roman «Auch wenn ich lache, muß ich weinen» grotesk, elegisch, allegorisch dargestellt. Sie erscheint als ein Exempel für die den einfachen, Menschen verachtende Politik. Closette fungieren als psychologische Metapher der Verdrängungen, aus denen die kritisierte Politik entsteht. Den Design-Produkten des Westens ( «Clo-o-Form ») wird das «Öko-Klo» des DDR-Installateurs Mischa Kafanke gegenübergestellt, mit dem er die Menschen-Scheiße sinnvoll zu entsorgen hofft. Die « Wiedervereinigung » ist nur einer der Krisenfälle der Weltpolitik, durch die Mischa, « der Gemischte » und daher amtlich nie eindeutig Beschreibbare, zu seiner Flucht aus dem vereinigten Deutschland nach Rußland und (über den Irak, Israel, Bosnien) in die USA gezwungen wird. Der Roman, der häufig auf literarische Muster verweist, ist als barocke Exempeldichtung konzipiert.

Dans le roman de Simmel "Auch wenn ich lache, muß ich weinen», la «réunification» est présentée à la fois sur un mode grotesque, élégiaque et allégorique. Elle apparait comme l'exemple d'une politique qui méprise l'être humain. Les WC jouent le rôle métaphorique des refoulements à l'origine de la politique critiquée. Aux produits du design occidental («Clo-o-Form») s'oppose le WC écologique de Mischa Kafanke, un plombier de la RDA, qui espère évacuer ainsi de manière impérieuse la Merde humaine. La «réunification» n'est pas seulement l'une des crises de la politique mondiale qui oblige Mischa à fuir l'Allemagne réunifiée pour aller en Russie, puis aux États-Unis en passant par l'Irak, Israël et la Bosnie. Mischa est, en effet, un «métis» qui n'a jamais d'identité clairement définissable sur le plan administratif. Le roman, qui se réfère souvent à des modèles littéraires, est conçu comme la poésie exemplaire baroque.

\section{AUTEUR}

\section{FRIEDBERT ASPETSBERGER}

Universität Graz 